06,11

\title{
Влияние концентрации La на кинетику индуцированного фазового перехода в прозрачной керамике $\mathrm{PbMg}_{1 / 3} \mathrm{Nb}_{2 / 3} \mathrm{O}_{3}-25 \mathrm{PbTiO}_{3}$
}

\author{
(C) Л.С. Камзина ${ }^{1}$, G. $L i^{2}$ \\ ${ }^{1}$ Физико-технический институт им. А.Ф. Иофрфе РАН, \\ 194021 Санкт-Петербург, Россия \\ ${ }^{2}$ Shanghai Institute of Ceramics, Chinese Academy of Sciences, \\ Shanghai, China \\ E-mail: askam@mail.ioffe.ru
}

Поступила в Редакцию 3 декабря 2019 г.

В окончательной редакции 3 декабря 2019 г.

Принята к публикации 3 декабря 2019 г.

\begin{abstract}
Изучено влияние концентрации La на кинетический характер индуцированного фазового перехода в сегнетокерамике $\mathrm{PbMg}_{1 / 3} \mathrm{Nb}_{2 / 3} \mathrm{O}_{3}-25 \mathrm{PbTiO}_{3}$. При температурах ниже температуры Фогеля-Фулчера исследованы изменения во времени диэлектрической проницаемости и оптического пропускания в электрических полях $0<E<6 \mathrm{kV} / \mathrm{cm}$. Обнаружено, что в электрическом поле в La-содержащей прозрачной керамике происходит резкое уменьшение диэлектрической проницаемости и оптического пропускания со временем, свидетельствующее об индуцированном фазовом переходе и увеличении доли сегнетоэлектрической фазы. Показано, что в недопированной La керамике изменения диэлектрической проницаемости при тех же полях существенно меньше. Обнаружено, что стабильность индуцированной фазы зависит от концентрации La: чем больше концентрация, тем меньше стабильность. Полученные результаты объясняются как разной степенью размытия фазового перехода, так и разными размерами полярных областей, зависящими от концентрации La.
\end{abstract}

Ключевые слова: сегнетоэлектричество, релаксоры, фазовые переходы, прозрачная керамика.

DOI: 10.21883/FTT.2020.04.49124.644

\section{1. Введение}

Монокристаллические твердые растворы $\mathrm{PbMg}_{1 / 3} \mathrm{Nb}_{2 / 3} \mathrm{O}_{3-x} \mathrm{PbTiO}_{3}$ (PMN-xPT) интенсивно исследуются последние два десятилетия как многообещающие материалы для актюаторов, сенсоров и других электромеханических устройств. Эти оксидные материалы являются также интересными и для нелинейных оптических применений. Однако изучение нелинейных оптических свойств сопряжено $\mathrm{c}$ определенными трудностями, связанными как с отсутствием высококачественных кристаллов больших размеров, пригодных для применений, так и с высокой стоимостью изготовляемых из них элементов. Использование керамики с высокой прозрачностью в сочетании с потенциально большей технологичностью и экономичностью изготовления и низкой стоимостью может устранить эти трудности и открыть большие возможности для применения. Действительно, Jiang [1] в 2005 г. приготовил прозрачную керамику PMN-xPT и сообщил о большой величине электрооптического эффекта в ней, однако не указал конкретный ее состав.

В наших работах [2-4] мы показали, что одним из наилучших составов прозрачной керамики, обладающих наивысшим значением квадратичных электрооптических (ЭО) коэффициентов $\left(R_{\mathrm{eff}}=66 \cdot 10^{16}(\mathrm{~m} / \mathrm{V})^{2}\right)$ и наименьшим полуволновым напряжением $(V \pi \sim 230 \mathrm{~V})$ среди всех релаксорных систем, является состав
PMN-25PT с добавкой 3\% La. Чтобы достичь высокой прозрачности, к твердым растворам PMN- $x$ PT добавлялся $\mathrm{La}^{3+}$. Среди кристаллических соединений наибольший квадратичный ЭО-эффект наблюдался в релаксорных монокристаллах PMN- $x$ PT с $x=13 \%$ $\left(R_{11}-R_{12}=32 \cdot 10^{16}(\mathrm{~m} / \mathrm{V})^{2}\right.$. Дальнейшее увеличение $x$ $(x>13 \%)$ приводит к уменьшению ЭО-эффекта. Такая картина наблюдается в соединениях PMN- $x$ PT без примеси La. Увеличение $x$ приводит к уменьшению доли релаксорных областей с большим квадратичным ЭО-эффектом. В составе PMN-25PT без примеси La доля релаксорных областей невелика и значительную часть объема занимают области сегнетоэлектрической фазы, обладающие спонтанным фазовым переходом из релаксорного в сегнетоэлектрическое состояние в отсутствие поля. По-видимому, ключевым моментом в большой величине ЭО-эффекта, наблюдаемой в соединениях PMN-25PT, является добавка ионов La.

Следовательно, введение ионов La не только увеличивает прозрачность керамики, но и влияет на электрооптические свойства.

В ряде работ исследовалось влияние La разной концентрации на диэлектрические свойства и доменную структуру в некоторых релаксорных соединениях. Так в работе [5] при изучении составов PMN-35PT, лежащих на морфотропной фазовой границе, было показано, что введение La разрушает дальний сегнетоэлектрический порядок, наблюдаемый в отсутствие $\mathrm{La}$, и приводит к 
тому, что полярные нанодомены становятся стабильной доменной структурой. Средний размер этих полярных областей уменьшается с увеличением концентрации La. Авторы работы [6] обнаружили, что допирование 5\% La керамических соединений PMN приводит к подавлению индуцированного электрическим полем фазового перехода в сегнетоэлектрическую фазу в этом материале.

Таким образом, в приведенных литературных данных и наших работах обнаружено большое влияние $\mathrm{La}$ на целый ряд физических свойств релаксоров. Тем интереснее исследовать влияние La на другие, еще не изученные свойства. Совсем нет сведений о влиянии $\mathrm{La}$ на кинетику процесса поляризации при индуцировании фазового перехода в электрическом поле в прозрачной сегнетокерамике PMN-25PT, являющейся прекрасным электрооптическим материалом.

Составы PMN-25PT относятся к релаксорам. Напомним, что необычные свойства релаксорных материалов связаны с присутствием сегнетоэлектрических наноразмерных областей ромбоэдрической симметрии (полярные нанообласти PNR), которые возникают при высокой температуре (температура Бернса), превышающей температуру максимума диэлектрической проницаемости. Эти полярные области, внедренные в неполярное окружение, пространственно ориентированы. При охлаждении увеличивается число и размеры полярных областей, увеличивается взаимодействие между ними, При некоторой температуре (температура замерзания, или температура Фогеля-Фулчера $T_{f}$ ) они замерзают. В замороженном релаксорном состоянии PNR приобретают слабую кинетику, но не образуют дальнего порядка. Свойства системы в таком неравновесном состоянии должны зависеть от времени. В электрическом поле происходит трансформация стекольного состояния в фазу с дальним порядком.

С этой целью в настоящей работе измерялись временные зависимости диэлектрической проницаемости $(\varepsilon)$ и оптического пропускания в прозрачной сегнетокерамике PMN-25PT, допированной 2 и $3 \% \mathrm{La}$, в различных электрических полях. Полученные результаты по диэлектрической проницаемости сравнивались с результатами, полученными для недопированной сегнетокерамики PMN-25PT.

\section{2. Исследуемые образцы и методика эксперимента}

Высокопрозрачная La-содержащая керамика PMN-25PT $(y / 75 / 25)$ с концентрацией La $y=2.3 \%$ приготавливалась с использованием необычного двухступенчатого метода спекания, описанного в наших работах $[3,4]$ и отличающегося от стандартного (типичного) одноэтапного метода горячего прессования [1]. Полученные образцы были высокого оптического качества. Недопированная сегнетокерамика PMN-25PT (0/75/25) приготавливалась по обычной керамической технологии.
Для оптических измерений применялся $\mathrm{He}-\mathrm{Ne}-$ лазер. Пропускание образцов PMN-25PT, допированных $3 \% \mathrm{La}(3 / 75 / 25)$, при комнатной температуре составляло $\sim 65 \%$ в области $600-2100 \mathrm{~nm}$. В образцах PMN-25PT, допированных $2 \% \mathrm{La}(2 / 75 / 25)$, наблюдалось сильное рассеяние.

Исследуемые образцы имели следующие характерные температуры: $2 / 75 / 25-T_{\max }=340 \mathrm{~K}, T_{f}=313 \mathrm{~K}$; $3 / 75 / 25-T_{\max }=325 \mathrm{~K}, \quad T_{f}=283 \mathrm{~K} ; \quad 0 / 75 / 25-$ $T_{\max }=373-377, T_{f}=364-368 \mathrm{~K}$.

При измерении временных зависимостей $\varepsilon$ и оптического пропускания образцы после отжига охлаждались без поля до фиксированной температуры, при которой к ним прикладывалось постоянное электрическое поле разной напряженности. После приложения электрического поля осуществлялась регистрация изменения диэлектрической проницаемости и оптического пропускания со временем. Измерения проводились непосредственно после отжига образцов. Универсальный измеритель Е7-11 использовался для диэлектрических измерений. Измерения $\varepsilon$ приводились на частоте $1 \mathrm{kHz}$.

\section{3. Экспериментальные результаты}

Диэлектрические свойства и доменная структура изучаемых в данной работе образцов 2/75/25 и 3/75/25 подробно исследовалась в нашей работе [7]. Было показано, что все исследованные образцы имеют широкий максимум диэлектрической проницаемости $(\varepsilon)$ и частотную дисперсию $\varepsilon$, свидетельствующую о релаксороподобном поведении. При охлаждении в электрическом поле на температурной зависимости $\varepsilon$ при $T<T_{\max \varepsilon}$ наблюдался скачок и уменьшение частотной дисперсии. Это свидетельствует о индуцированном полем фазовым переходом из релаксорного состояния в сегнетоэлектрическое состояние с дальним порядком или в смешанную фазу. Чем больше содержание La, тем более размыт фазовый переход и менее выражен индуцированный фазовый переход. Разница в релаксорном поведении в исследуемых соединениях связана с разным числом и размерами полярных областей и их кинетикой. Увеличение содержания La усиливает релаксорные характеристики, или, другими словами, увеличивает энергетический барьер, который препятствует индуцированному фазовому переходу.

Для более наглядной картины этого различия на рис. 1 приведена схематическая иллюстрация доменной конфигурации для прозрачной керамики 2/75/25 и 3/75/25, и ее изменение в электрическом поле, опубликованная нами в работе [7]. Из рисунка видно, что полярные области в керамике 2/75/25 существенно больше по размерам, чем в керамике $3 / 75 / 25$. В электрическом поле их размеры увеличиваются, объем кубической перовскитовой фазы значительно уменьшается, и поведение керамики 2/75/25 больше похоже на поведение нормального сегнетоэлектрика с дальним порядком. Из этого рисунка понят- 
$2 / 75 / 25$

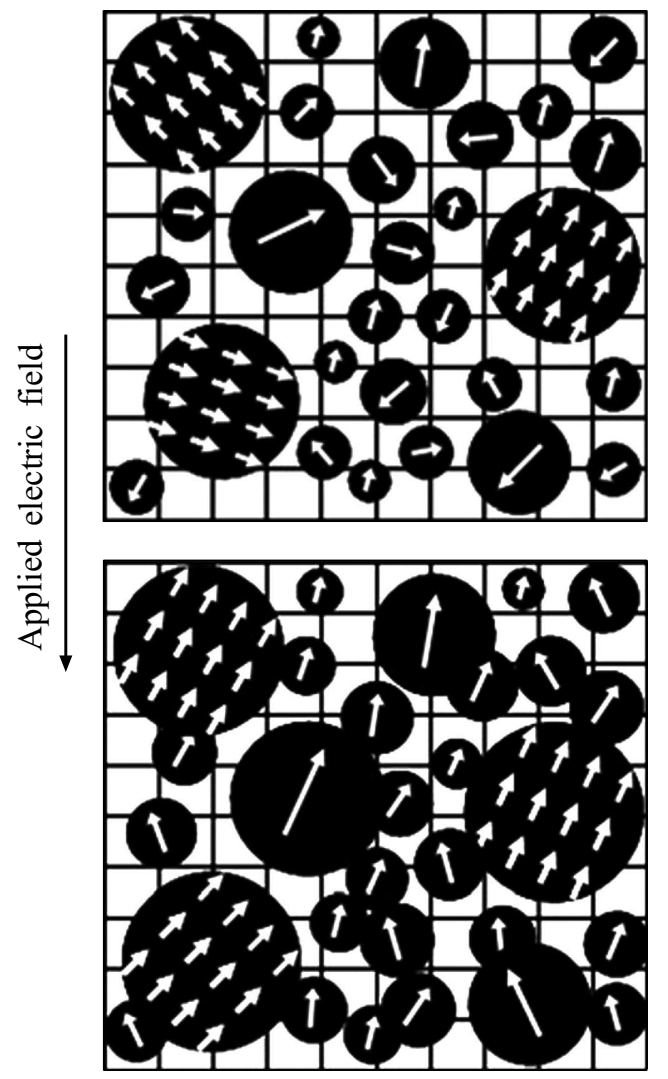

$3 / 75 / 25$
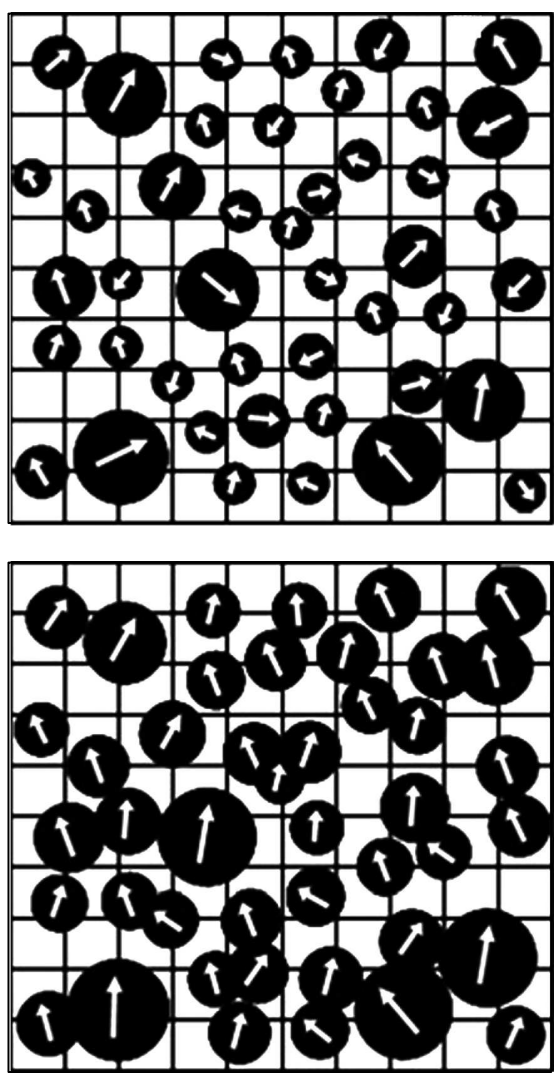

Рис. 1. Схематическая иллюстрация доменной конфигурации для керамики PMN-25PT, допированной La. Белые квадраты относятся к перовскитовой фазе, черные кружки со стрелками внутри - к полярным областям.

но, что большое рассеяние, наблюдаемое в керамике 2/75/25, связано с тем, что большие размеры полярных областей становятся сопоставимыми с длиной волны света.

В работе [8] сообщалось, что ромбоэдрическая фаза является единственной фазой при комнатной температуре для соединений PMN-25PT. При дальнейших исследованиях этих образцов при комнатной температуре в работе [9] обнаружено существование, кроме ромбоэдрической, еще и моноклинной $M_{A}$-фазы. В неполяризованных кристаллах при приложении электрического поля вдоль [001] наблюдался переход $R-M_{A}-T-C[10,11]$. Переход в тетрагональную фазу может происходить только в больших электрических полях и высоких температурах.

На рис. 2,3 представлены временные зависимости изменений диэлектрической проницаемости $\left(\varepsilon / \varepsilon_{0}\right)$ в разных электрических полях для керамики: 2/75/25 (рис. 2) и $3 / 75 / 25$ (рис. 3), где $\varepsilon_{0}$ - диэлектрическая проницаемость до приложения поля, $\varepsilon$ - диэлектрическая проницаемость в разные моменты времени. Поле прикладывалось к образцу в течении 600-800 s, после чего выключалось. Кривые рис. 2 сняты при комнатной температуре, а рис. 3 при $277 \mathrm{~K}$. Это связано с тем, что температура Фогеля-Фулчера для керамики 3/75/25 (рис. 3) находится ниже комнатной. Видно, что поля до $1.5 \mathrm{kV} / \mathrm{cm}$ не приводят к существенным изменениям $\varepsilon$ в керамике 2/75/25 (кривые 1,2 рис. 2). Более быстрое и заметное изменение $\varepsilon$ наблюдается в электрических полях, превышающих $1.5 \mathrm{kV} / \mathrm{cm}$. За короткий промежуток времени (кривые $3-5$ рис. 2) происходит резкое уменьшение $\varepsilon$, свидетельствующее об индуцированном фазовом переходе и увеличении доли сегнетоэлектрической фазы. Можно предположить, что столь сильное уменьшение величины $\varepsilon$ связано с уменьшением числа межфазных границ, переходом части ромбоэдрических областей в области моноклинной фазы и возникновением в части объема образца макродоменного состояния. В полях выше коэрцитивного поля $\left(E_{\text {коэр. }} \sim 4 \mathrm{kV} / \mathrm{cm}\right)$, ромбоэдрические домены, скорее всего, подвергаются моноклинному искажению типа $M_{A}$ и поворачиваются к тетрагональным доменам.

В отличие от керамики 2/75/25, в составе 3/75/25 в поле $1.5 \mathrm{kV} / \mathrm{cm}$ уже происходят достаточно заметные изменения величины $\left(\varepsilon / \varepsilon_{0}\right)$. Как видно из рис. 1 в этом составе керамики полярные области имеют меньшие размеры и, следовательно, образец более чувствителен к приложенному полю. Для индуцирования упорядоченного состояния в электрическом поле нужны меньшие поля. 


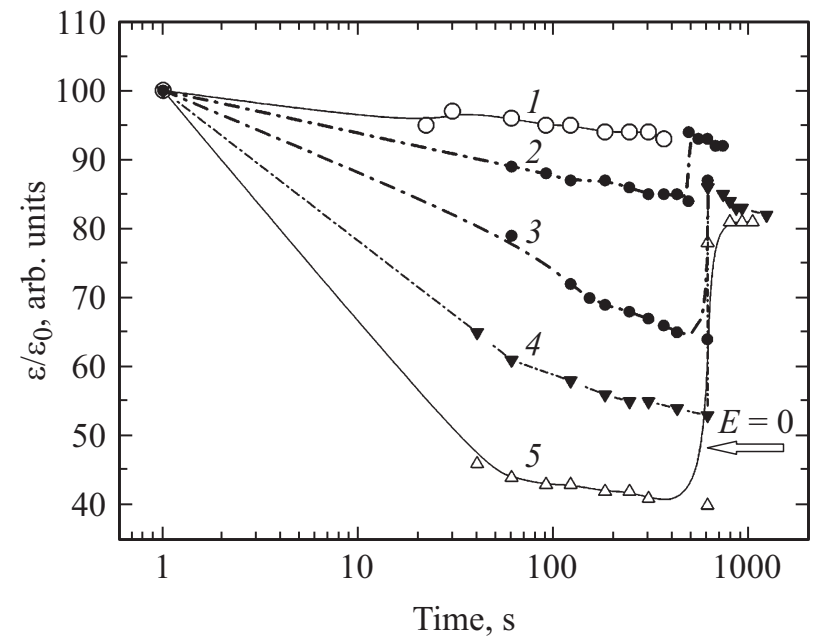

Рис. 2. Относительные изменения диэлектрической проницаемости $\left(\varepsilon / \varepsilon_{0}\right)\left(\varepsilon_{0}-\right.$ величина в нулевом поле) со временем при температуре $300 \mathrm{~K}$ в прозрачной керамике $2 / 75 / 25$ в разных электрических полях $(E, \mathrm{~V} / \mathrm{cm}): 1-1,2-1.5,3-2,4-3$, $5-5$.

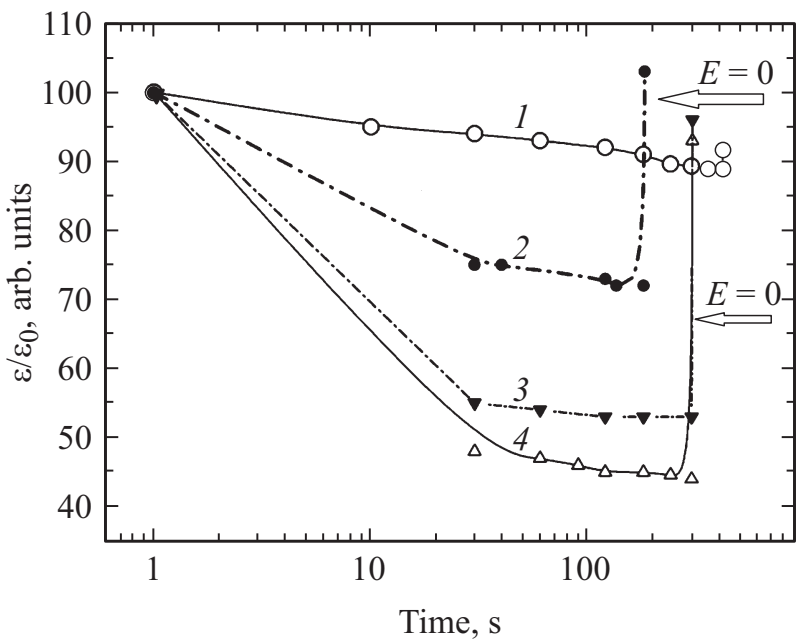

Рис. 3. Относительные изменения диэлектрической проницаемости $\left(\varepsilon / \varepsilon_{0}\right)$ со временем при температуре $277 \mathrm{~K}$ в прозрачной керамике $3 / 75 / 25$ в разных электрических полях $(E, \mathrm{kV} / \mathrm{cm})$ : $1-1,2-1.5,3-3,4-5$.

Выключение электрического поля приводит к скачкообразному возрастанию величины $\varepsilon$ (рис. 2 , кривые $2-5$, рис. 3 кривые $2-4$ ). В образцах $2 / 75 / 25$ (рис. 2) ее величина немного меньше, чем до приложения поля, т.е. остается небольшая доля заполяризованной фазы (5-10\%). Это может быть связано с возвращением не всего объема кристалла, а только определенной доли заполяризованных областей в первоначальное состояние. Заметим, что доля заполяризованной фазы в образцах 2/75/25 после выключения поля зависит от предварительно приложенного поля.

В образцах 3/75/25 (рис. 3) после выключения электрического поля величина $\varepsilon$ возрастает до первоначаль- ного значения. Эти данные согласуются с результатами работ $[5,6]$. Добавка La приводит к частичному замещению ионов $\mathrm{Pb}$ ионами $\mathrm{La}$ и появлению $\mathrm{Pb}$-вакансий в состоянии $A$ перовскита. Эти вакансии понижают связь между октаэдрами $\mathrm{BO}_{6}$, ослабляют взаимодействие между дипольными моментами и препятствуют возникновению макроскопического дальнего порядка. Чем больше содержание $\mathrm{La}$, тем больше размыт фазовый переход и тем менее стабильна индуцированная в поле фаза с дальним порядком.

Это подтверждают результаты временных зависимостей $\varepsilon$ для керамики $(0 / 75 / 25)$, приведенные на рис. 4 . Во-первых, изменения величины $\varepsilon$ в тех же полях, что и на рис. 2 и 3 , существенно меньше. В керамике 0/75/25 фазовый переход размыт меньше, размеры полярных областей больше, межфазных границ меньше и, следовательно, доля релаксорной стеклоподобной фазы незначительна. Во-вторых, индуцированная фаза, скорее всего моноклинная, является более стабильной после снятия поля (кривая 3), чем для керамик с добавкой $\mathrm{La}$ (рис. 2, 3).

Результаты измерений оптического пропускания в электрических полях не противоречат диэлектрическим измерениям. На рис. 5 (кривые $1-3$ ), в качестве примера, приведены временные зависимости оптического пропускания в разных электрических полях для состава $2 / 75 / 25$. Также, как и в случае диэлектрических измерений, за короткий промежуток времени после приложения поля, большего некоторого критического поля, наблюдается резкое уменьшение интенсивности оптического пропускания. Уменьшение пропускания обусловлено ростом электрически индуцированных полярных областей или доменов (см. рис. 1). Возникновение в поле частично упорядоченной сегнетоэлектрической структуры или доменов больших размеров может приводить,

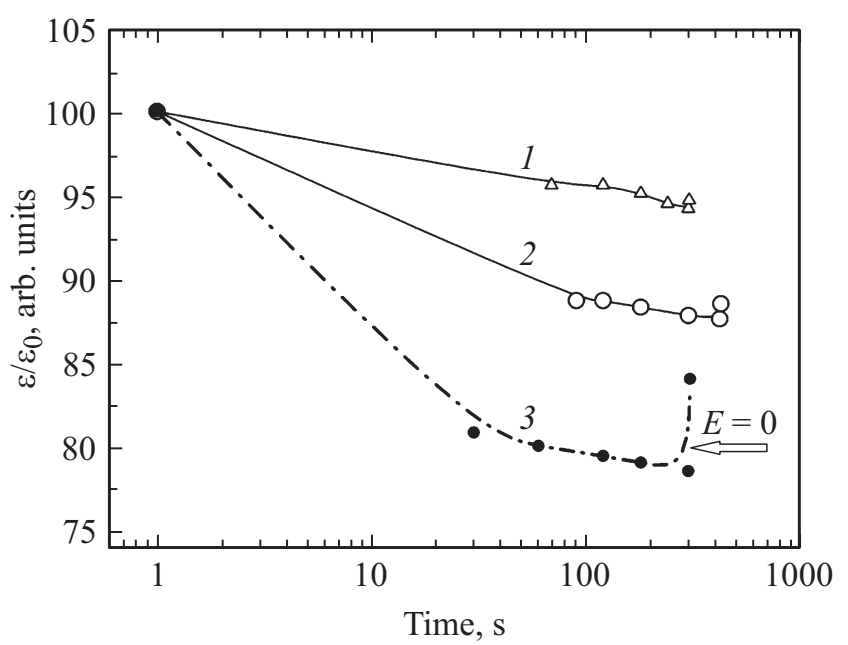

Рис. 4. Зависимость диэлектрической проницаемости $\left(\varepsilon / \varepsilon_{0}\right)$ от времени при температуре $300 \mathrm{~K}$ в недопированной керамике $\mathrm{PMN}-25 \mathrm{PT}$ в разных электрических полях $(E, \mathrm{kV} / \mathrm{cm}): 1-2$, $2-4,3-6$. 
соответственно, к большим доменным стенкам, которые рассеивают падающий свет и уменьшают пропускание. Аналогичная зависимость наблюдалась и для керамики $3 / 75 / 25$.

На рис. 6 представлены зависимости изменения $\varepsilon$ от напряженности электрического поля для керамик 0/75/25 (кривая 1) и 3/75/25 (кривая 2). Кривая 1 измерялась при комнатной температуре, а кривая 2 при $277 \mathrm{~K}$. Каждое поле прикладывалось к образцам в течение $2 \mathrm{~min}$. Данные рисунка подтверждают полученные выше результаты о влиянии La на стабильность возникающей в поле сегнетоэлектрической фазы с дальним порядком.

Ранее нами в работе [4] уже приводилась зависимость диэлектрической проницаемости от напряжен-

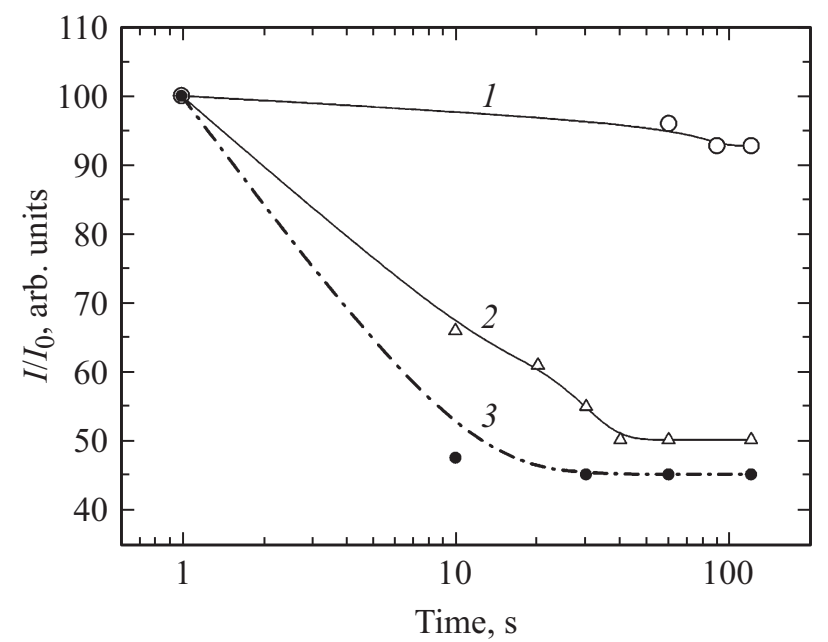

Рис. 5. Изменения со временем относительной величины оптического пропускания $\left(I / I_{0}\right)\left(I_{0}-\right.$ величина в нулевом поле) в прозрачной керамике $2 / 75 / 25$ в разных электрических полях $(E, \mathrm{kV} / \mathrm{cm}): 1-2 ; 2-3 ; 3-4$.

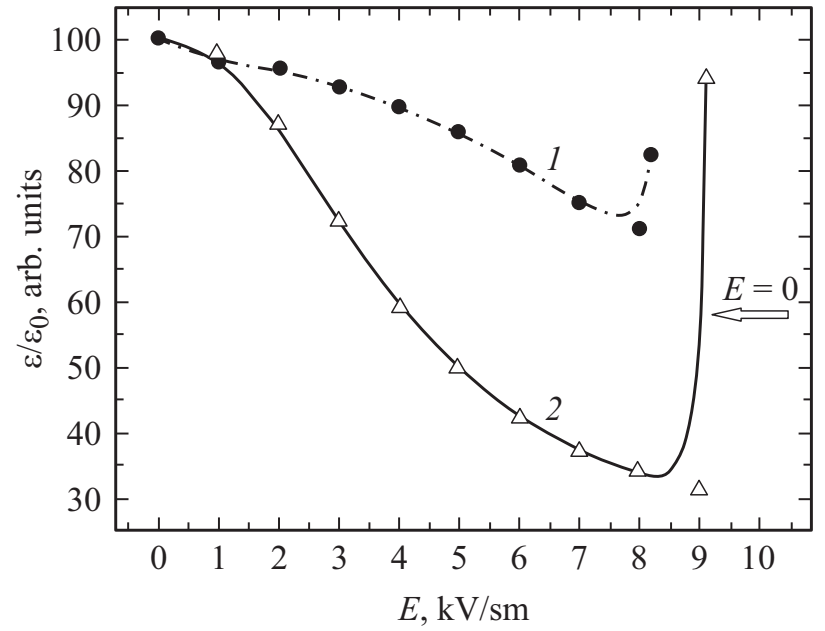

Pис. 6. Зависимость диэлектрической проницаемости от напряженности электрического поля для недопированной керамики PMN-25PT при $300 \mathrm{~K}$ (кривая 1) и прозрачной керамики $3 / 75 / 25$ при $277 \mathrm{~K}$ (кривая 2). Каждое поле прикладывалось в течение 2 минут.

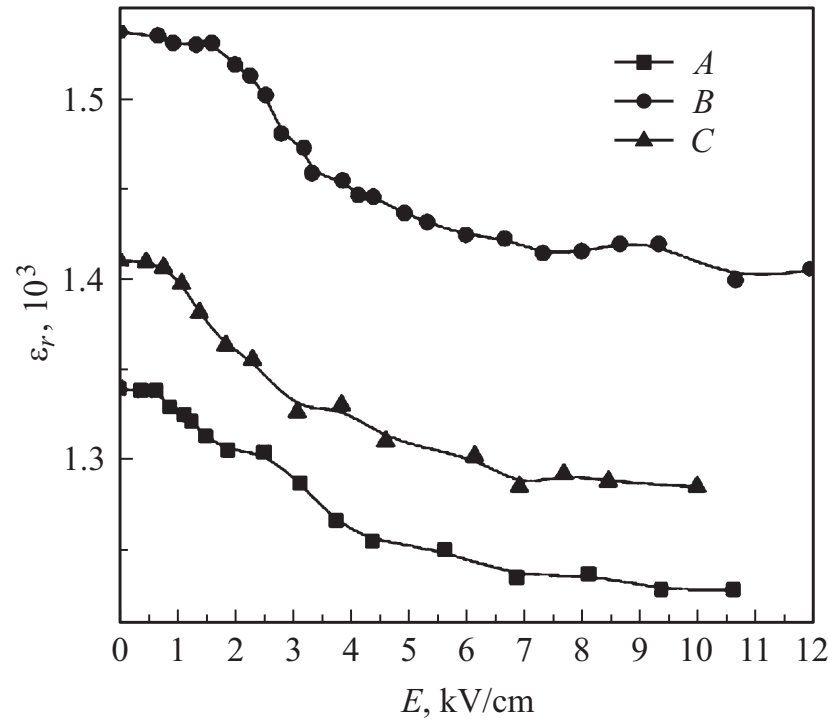

Рис. 7. Зависимость диэлектрической проницаемости от напряженности электрического поля при $300 \mathrm{~K}$ в 3-х образцах прозрачной керамики PMN-25PT, допированной 3\% La (3/75/25), спеченных при разных температурах: $A-1150^{\circ} \mathrm{C}$, $B-1200^{\circ} \mathrm{C}, C-1230^{\circ} \mathrm{C}$. Каждое поле прикладывалось в течение 1 минуты [4].

ности электрического поля для прозрачной керамики 3/75/25. Эти зависимости представлены на рис. 7 для трех образцов 3/75/25, которые спекались при разных температурах. Видно, что изменения $\varepsilon$ в электрическом поле на рис. 6 сушественно больше, чем на рис. 7. Причины такого различия понятны. Во-первых, электрическое поле на рис. 6 прикладывалось к образцам при комнатной температуре. Эта температура выше температуры Фогеля-Фулчера для состава 3/75/25 и при этой температуре образец уже не находится в стекольной релаксорной фазе, что приводит к меньшей зависимости $\varepsilon$ от величины поля. Во-вторых, поле к образцам на рис. 6 прикладывалось в течение $1 \mathrm{~min}$, а стабильное сегнетоэлектрическое состояние, как видно из рис. 3, возникает при более длительном приложении поля.

\section{4. Заключение}

В данной работе впервые изучены изменения со временем диэлектрической проницаемости и оптического пропускания в разных электрических полях как в La coдержащих керамических соединениях PMN-25PT, так и в недопированном PMN-25PT. Обнаружено, что при температурах ниже температуры Фогеля-Фулчера, в электрических полях, больших некоторого порогового поля, в La содержащей керамике происходит резкое уменьшение $\varepsilon$ и оптического пропускания, свидетельствующее об индуцированном фазовом переходе и увеличении доли сегнетоэлектрической фазы. В недопированной керамике изменения $\varepsilon$ при тех же полях существенно меньше. 
Показано, что поляризованная фаза, возникшая в электрическом поле, не является полностью стабильной. Об этом свидетельствует скачкообразное увеличение диэлектрической постоянной после выключения поля.

Обнаружено, что чем больше содержание La, тем больше размыт фазовый переход и меньше стабильность возникающей в поле сегнетоэлектрической фазы с дальним порядком. В недопированной керамике и в керамике, содержащей $2 \% \mathrm{La}$, величина $\varepsilon$ не возвращаются к исходному значению до приложения поля, т.е. остается небольшая доля заполяризованной фазы. Это подтверждает факт не полной, а только частичной поляризации образца. В керамике, содержащей 3\% La, после выключения электрического поля величина $\varepsilon$ возрастает до первоначального значения.

\section{Конфликт интересов}

Авторы заявляют, что у них нет конфликта интересов.

\section{Список литературы}

[1] H. Jiang, Y.K. Zou, Q. Chen, K.K. Li, R. Zhang, Y. Wang, H. Ming, Z. Zheng. Proc. SPIE 5644, 380 (2005).

[2] L.S. Kamzina, Ruan Wei , Jiangto Zeng, Guorong Li. ФTT 53, 8, 1530 (2011).

[3] Л.С. Камзина, Ruan Wei, G. Li, J. Zeng, A. Ding. ФТT 52, 10, 1999 (2010).

[4] Wei Ruan, Guorong Li, Jiangtao Zeng, Jianjiang Bian, L.S. Kamzina, Huarong Zeng, Liaoying Zheng, Aili Ding. J. Am. Cer. Soc. 93, 8, 2128 (2010).

[5] Surya M. Gupta, Dwight Viehland. J. Appl. Phys. 80, 5875 (1996).

[6] Xiaohui Zhao, Weiguo Qu, Hui He, Naratip Vittayakorn, Xiaoli Tan. J. Am. Ceram. Soc. 89, 1, 202 (2006).

[7] Wei Ruan, Guorong Li, Jiangtao Zeng, L.S. Kamzina, Huarong Zeng, Kunyu Zhao, Liaoying Zheng, Aili Ding. J. Appl. Phys. 110, 074109 (2011).

[8] O. Noblanc, P. Gaucher, G. Calvarin. J. Appl. Phys. 79, 4291 (1996).

[9] F. Bai, N. Wang, J. Li, D. Viehland, P.M. Gehring, G. Xu, G. Shirane. J. Appl. Phys. 96, 1620 (2004).

[10] R. Chien, V.H. Schmidt, C.S. Tu, L.W. Hung, H. Luo. Phys. Rev. B 69, 172101 (2004).

[11] Matthew Davis, Dragan Damjanovic, Nava Setter. Phys. Rev. B 73, 014115 (2006).

Редактор Т.Н. Василевская 